\title{
Canales y márgenes de ComercializaCión de leche bovina en la PARroquia Guasaganda, Cantón La Maná, Cotopaxi, Ecuador
}

\author{
Trading channels and margins of bovine milk in Guasaganda parish, La Mana canton, Cotopaxi, Ecuador
}

${ }^{\bullet}$ Emma Torres Navarrete ${ }^{1}$, Marco Ludeña Vidal², Fabi Villagómez Zambrano ${ }^{2}$, Guadalupe Murillo Campuzano ${ }^{3}$, Adolfo Sánchez Laíño ${ }^{1}$, Yenni Torres Navarrete ${ }^{1}$

${ }^{1}$ Facultad de Ciencias Pecuarias, Universidad Técnica Estatal de Quevedo. Campus Finca Experimental "La Maria” km 7 vía Quevedo-El Empalme.EC.120501. Mocache,Ecuador. ${ }^{\circ}$ etorres@uteq.edu.ec; arsanchez@uteq.edu.ec; ytorres@uteq.edu.ec

${ }^{2}$ Facultad de Ciencias Agrarias, Universidad Técnica Estatal de Quevedo. Campus Ing. Manuel Haz Álvarez, km 1.5 vía a Santo Domingo de los Tsáchilas.EC.120501.Quevedo,Ecuador. marcoproesa@hotmail.com; abenllagomez12@yahoo.com ${ }^{3}$ Unidad de Estudios a Distancia, Universidad Técnica Estatal de Quevedo. Campus Ing. Manuel Haz Álvarez, km 1.5 vía a Santo Domingo de los Tsáchilas.EC.120501.Quevedo,Ecuador. lupita_pilar11@hotmail.com

RESUMEN

$\mathrm{E}_{\mathrm{in}}^{1}$ objetivo de la investigación fue proporcionar información sobre las características del mercadeo de leche bovina en la parroquia Guasaganda, cantón La Maná, provincia de Cotopaxi, Ecuador. La metodología consistió en la identificación de los canales de comercialización y establecimiento de los márgenes de comercialización en la zona. Se inició con la identificación del origen del producto, sus características, y la observación sistemática de todos los procesos que incluyeron cambios en su forma y propiedad hasta llegar al consumidor final. Se diseñó y aplicó una encuesta a los diferentes agentes que realizan funciones de comercialización; productores (P), acopiadores (A), industria pasteurizadora (IP) y artesanal (IA) y, detallistas (D). Se obtuvo el margen bruto de comercialización (MBC), margen neto de comercialización (MNC) y la participación del productor (PDP). Además, se obtuvieron costos de producción y rentabilidad de un litro de leche a nivel de productores. Los resultados muestran la comercialización de tres productos: leche cruda, fluida y queso. Se determinaron dos canales de comercialización: el canal corto $40 \%$ de pequeños productores: productor-consumidor final) y el canal convencional $(100 \%$ de medianos y grandes productores y $60 \%$ de pequeños productores: productor-acopiador rural - industria pasteurizadora detallista - consumidor final (leche fluida); productor - acopiador rural - industria artesanal de queso detallista - consumidor final). Los márgenes obtenidos en la comercialización de leche fluida, en promedio, son: MBC (60\%), PDP (40\%), y MNC (38.7\%) y los márgenes en la comercialización de queso en promedio son: $\mathrm{MBC}$ (40.34\%), PDP (59.66\%), y MNC (29.8\%).

Palabras clave: mercadeo, leche bovina, agentes de comercialización.

Recibido: 22-agosto-2013. Recibido en forma corregida: 18-enero-2014.

Aceptado: 23-abril-2014.

Publicado como ARTÍCULO CIENTÍFICO en Ciencia y Tecnología 7(2): 1-8

Julio-Diciembre de 2014

ISSN 1390-4051 impreso; ISSN 1390-4043 electrónico

(C) Ciencia y Tecnología. UTEQ. Quevedo-Ecuador

\section{Abstract}

$T$ he aim of the research was to provide information on the characteristics of bovine milk marketing in Guasaganda parish, La Mana canton, Cotopaxi province, Ecuador. The methodology consisted in identifying marketing channels and establishing marketing margins in the area. It began with the identification of the origin of the product, its features, and systematic observation during all processes involving changes in form and properties until it reaches the final consumer. A survey of the different agents that perform marketing: producers (P), intermediaries (A), pasteurizer industry (IP) and artisan (IA) and retailers (D). Gross marketing margin $(\mathrm{MBC})$, net marketing margin $(\mathrm{MNC})$ was designed and implemented and the percentage of producer participation (PDP) was obtained. Besides production costs and profitability of a liter of milk at producer level were obtained. The results show the marketing of three products: raw milk, fluid milk and cheese. Two main trading channels were determined: short channel (40\% of smallholders: producer-final consumer) and conventional channel $(100 \%$ of medium and large producers and $60 \%$ of small producers: producer - rural gatherer - pasteurizer industry - retailer- final consumer two outlets were determined - final consumer (fluid milk) - producer - rural gatherer - cheese handicraft industry - retailer - final consumer). The gross trading margins of the fluid milk, on average, were: MBC $(60 \%)$, PDP (40\%) and MNC (38.7\%) and the gross trading margins of cheese, on average, were: $\mathrm{MBC}$ (40.34\%), PDP (59.66\%) and MNC (29.8\%).

Key words: marketing, bovine milk, marketing agents. 


\section{INTRODUCCIÓN}

$\mathrm{L}$ a leche constituye uno de los alimentos más completos y recomendados en la dieta básica de los seres humanos, razón por la que forma parte de las estrategias de seguridad alimentaria (FAO, 1999). $\mathrm{Su}$ creciente consumo en el mundo se debe a que este producto es una fuente alimenticia económicamente más accesible y de mayor demanda en la población de bajos recursos (García et al., 2005). Según Padilla 2005, el consumo per cápita anual de leche en Ecuador es $110 \mathrm{~L}_{\text {habitante }}{ }^{-1}$, cifras inferiores a las de Argentina (220 L/habitante L $^{-1}$ y Uruguay (270 L habitante $^{-1}$ ) y a las recomendadas por OMS (270 $\mathrm{L}_{\text {habitante }}{ }^{-1}$ ).

FAO y FIL (2012) calculan que alrededor de 150 millones de hogares el mundo se dedican a la producción de leche. En la mayoría de los países en desarrollo, la leche es producida por pequeños agricultores y la producción lechera contribuye a los medios de vida, la seguridad alimentaria y la nutrición en los hogares. La leche produce ganancias relativamente rápidas para los pequeños productores y es una fuente importante de ingresos en efectivo.

La producción total de leche a nivel mundial correspondiente al año 2011 fue de 730.1 millones de toneladas métricas, lo que representó un crecimiento del $2.31 \%$ con respecto al año precedente, por su parte, la producción lechera de América Latina y el Caribe han mostrado un mayor grado de dinamismo que el conjunto de la producción mundial en los últimos 20 años, mientras que esta última aumentó un 31\%, la de Sudamérica lo hizo en $108 \%$ y, América Central y México en $70 \%$. Sólo la producción de la región del Caribe ha sido menos dinámica que la del resto del mundo, ya que sólo aumentó un $8 \%$ en los últimos 20 años (FAO-FEPALE, 2012).

Las producciones presentadas en el Gráfico 1 son las reportadas por FAO-FEPALE (2012) que surgen de la información oficial que aporta el sector público de cada país; se muestra la producción de leche de 19 países de América Latina y El Caribe con 83.217 millones de litros en el año 2011. La producción de leche en esta región está concentrada, y a tono con las diferencias de tamaño geográfico de los países, siendo el principal productor (Brasil) con el 39\% de la producción, el rango de precios del litro de leche en la región va de 0.37 a 0.53 dólares.

La misma fuente sostiene que existirían en los 19 países analizados unos 3.15 millones de productores de leche, lo que permite observar la importancia de la actividad ganadera en términos que van más allá de la contribución al producto o a la alimentación, sino concretamente por la ocupación del terreno y el empleo que generan.
En este sentido, el sector lechero proporciona más empleo por unidad de producción de leche en los países en desarrollo que en los países desarrollados. Esto se debe principalmente a que los países desarrollados tienen sistemas de producción con un mayor uso de tecnología y un menor empleo de mano de obra. Mientras que, en los países en desarrollo la producción lechera a pequeña escala orientada al mercado genera empleo en la explotación y aumenta los ingresos agropecuarios, además de generar empleos fuera de la finca y oportunidades de ingresos en la recolección, comercialización y procesamiento de la leche. De igual manera ofrece a los pequeños agricultores mayores utilidades que los cultivos y genera más oportunidades de empleo que otras cadenas de valor del sector alimentario.

FAO-FEPALE (2012), presentan información de los países que cuentan con mayor cantidad de productores de leche: Brasil (1.35 millones), Colombia (495,000), Ecuador $(301,500)$, Cuba (estimados 300,000) y México $(259,000)$. También tienen cantidades significativas de productores de leche países como Nicaragua $(120,000)$ y Perú $(100,000)$.

En Ecuador la actividad ganadera-lechera ha sido por tradición uno de los medios de subsistencia más antiguos, ya que constituye fuentes de alimentación dentro de las unidades de producción y de ingresos con productos elaborados artesanalmente fuera de ellas (queso, mantequilla, yogurt, y otros). Tradicionalmente, este rubro productivo se ha concentrado en la región Interandina, donde se ubican los mayores hatos lecheros (73\%), mientras que en la Costa se cuenta con un 19\% y en el Oriente y Región Insular con el 8\% de la producción nacional (Torres et al., 2014). Un 75\% de la producción bruta de leche cruda está destinada al consumo humano e industrial (SICA-MAGAP, 2000).

Según SICA-MAGAP (2000), la provincia de Cotopaxi tenía el $17.8 \%$ del total de ganado productor del país, estableciéndose además un promedio de producción de $5.9 \mathrm{~L} \mathrm{día}^{-1}$, cuando en el resto del país promediaba $4.4 \mathrm{~L}_{\text {vaca.día }}{ }^{-1}$, demostrándose que en la provincia este rubro productivo es muy trascendente.

La producción de ganado lechero para los habitantes de la parroquia Guasaganda perteneciente al cantón La Maná, provincia de Cotopaxi es de suma importancia, ya que además de ser un producto básico de la canasta familiar, tiene un alto valor nutritivo y es un gran generador de empleo entre las familias campesinas. Se conoce que la zona es eminentemente ganadera, sin embargo, existe escaso conocimiento de los sistemas de producción y comercialización de leche, lo que causa incertidumbre con respecto a la rentabilidad que genera 
esta actividad a cada uno de los agentes que participan en el proceso de comercialización por lo que se planteó como objetivo determinar los márgenes y canales de comercialización de leche producida en dicha parroquia.

\section{Materiales y Métodos}

L a investigación se realizó en la parroquia Guasaganda, cantón La Maná provincia de Cotopaxi (Figura 1), su ubicación, en un punto de transición entre la Sierra y la Costa ecuatoriana le confieren características especiales en cuanto a su relieve, biodiversidad, hidrografía y clima. La zona bajo estudio comprende $225 \mathrm{~km}^{2}$ de superficie, localizada a $20 \mathrm{~km}$. de La Maná, a $79^{\circ} 29^{\prime}$ de longitud Este y $1^{\circ} 06^{\prime}$ de latitud Sur, a una altura de $600 \mathrm{msnm}$, clima húmedo tropical, suelo franco-arenoso y arcilloso, características que propician el desarrollo de los pastos Gramalote (Axonopus dilatatum), Miel (Paspalum dilatatum), Brachiarias (Brachiaria decumbens), favoreciendo el crecimiento y desarrollo del ganado vacuno. La temperatura oscila entre 23 a $24.5^{\circ} \mathrm{C}$, humedad relativa de 75 a $84.5 \%$, heliofanía de 894 horas luz año, la etapa de lluvia en la zona es prolongada desde diciembre hasta julio, con una duración de siete meses, mientras que el resto del tiempo se caracteriza por leves precipitaciones, siendo el promedio anual de 2,100 a 2,300 $\mathrm{mm}$.

Para la ejecución de la investigación se utilizó la técnica de la encuesta directa a los agentes participantes en la comercialización de leche (productor, acopiador, industria pasteurizadora $\mathrm{y}$ artesanal, detallista $\mathrm{y}$ consumidor final), con estructuración de tipo cuanticualitativo y una dimensión temporal de tipo transversal, es decir los datos se tomaron en un corte de tiempo (Torres et al., 2013).

Para determinar el tamaño de la muestra de productores de leche se aplicó el muestreo aleatorio simple, sobre el cual todos y cada uno de los individuos de la población tienen la misma oportunidad de ser seleccionados como parte de la muestra (SantoyoCortés et al., 2000), la ecuación aplicada fue:

$$
n=\frac{(N)\left(Z^{2} \infty / 2\right)(p n)(q n)}{(N-1)\left(d^{2}\right)+\left(Z^{2} \infty / 2\right)(p n)(q n)}
$$

Donde:

$\mathrm{n}=$ tamaño de la muestra.

$\mathrm{N}=600$ (población total o universo).

$\mathrm{d}=10 \%$ (error muestral).

$\mathrm{Z} 2 \mathrm{o}^{2} \mathrm{2}=1.69$ (Coeficiente de confianza o confiabilidad). pn $=0.5$ (probabilidad de éxito o aceptación).

$\mathrm{qn}=0.5$ (probabilidad de fracaso o rechazo).

Al aplicar la fórmula se determinó que $\mathrm{n}=152$. con una población de 600 ganaderos en la parroquia Guasaganda. Posteriormente se estratificó en tres categorías de acuerdo al número de animales que poseían (Cuadro 1): grupo 1 (54 productores pequeños) de 10 a 65 bovinos; grupo 2 (53 productores medianos) de 66 a 160 bovinos; grupo 3 (45 productores grandes) 161 bovinos en adelante. La selección de las unidades de muestreo se realizó extrayendo aleatoriamente las unidades de la población a través de la tabla de números aleatorios.

Para determinar los márgenes de comercialización se entrevistó a todos los acopiadores (5) de leche de la zona de estudio.

En el desarrollo de la investigación se siguieron las siguientes etapas: 1) Reconocimiento y aceptación del grupo de trabajo en la comunidad para la identificación de los productores y acopiadores, 2) Diseño y aplicación

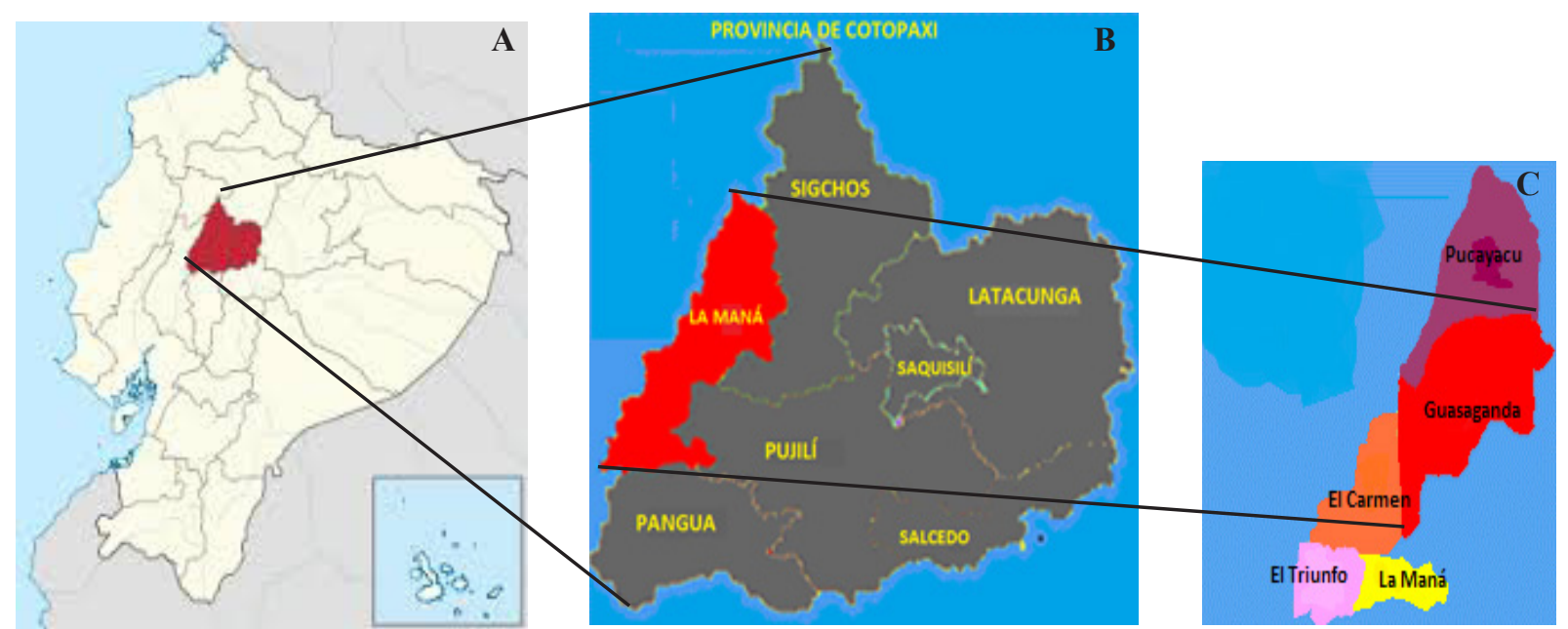

Figura 1. Localización del área de de estudio: provincia de Cotopaxi (A), cantón La Maná (B), y parroquia Guasaganda (C) 
Cuadro 1. Estratificación de productores en 17 localidades de la parroquia Guasaganda, La Maná. Prov. de Cotopaxi

\begin{tabular}{llccc}
\hline $\mathbf{N}^{\mathbf{0}}$ & Sectores & \multicolumn{3}{c}{ Pequeños Medianos Grandes } \\
\hline 1 Villa verde & 5 & 3 & 1 \\
2 & El tesoro & 1 & 1 & - \\
3 San Antonio & 3 & 1 & 1 \\
4 El tigre & 4 & 3 & 2 \\
5 El copal & 9 & 4 & 5 \\
6 Capilla chica & 5 & 2 & 4 \\
7 & San Cristóbal & 1 & 3 & 6 \\
8 La josefina & 1 & 3 & 1 \\
9 & La Florida & 3 & 7 & - \\
10 & 21 de Noviembre & 4 & 1 & 4 \\
11 La playa & 2 & 2 & 8 \\
12 & El triunfo & 3 & 3 & - \\
13 & Yanayacu & 5 & 5 & 5 \\
14 & San Marcos & 2 & 5 & 1 \\
15 & Juan Cabo & 4 & 3 & 3 \\
16 & Los Laureles & 1 & 5 & 2 \\
17 & San Vicente de los & 1 & 2 & 2 \\
\hline Tingos & & $\mathbf{5 4}$ & $\mathbf{4 5}$ \\
\hline
\end{tabular}

de cuestionarios para la obtención de información, 3) Identificación de los canales de comercialización, 4) Captura y análisis de la información y, 5) Obtención de los márgenes de comercialización mediante la metodología propuesta por Espinosa et al. (2008) utilizando las siguientes fórmulas:

Margen bruto de comercialización (MBC)

$M B C=\frac{\text { Precio al consumidor }- \text { Precio al productor }}{\text { Precio al consumidor }} \times 100$

Margen del productor (PDP)

$P D P=\frac{\text { Precio al productor }}{\text { Precio pagado por el consumidor }} \times 100$

Margen neto de comercialización (MNC)

MNC $=\frac{\text { Margen bruto }- \text { Costo de mercado }}{\text { Precio pagado por el consumidor }} \times 100$
Margen bruto de comercialización del acopiador rural $(\mathrm{MBC}-\mathrm{AR})$

$M B C A R=\frac{\text { Precio de venta }- \text { Precio de compra }}{\text { Precio pagado por el consumidor }} \times 100$

Para identificar los canales de comercialización se siguió el método directo (monitoreo del canal de comercialización de la leche, desde su salida de la finca hasta el consumidor final con el propósito de determinar el número de agentes participantes, precios y costos a cada nivel de comercialización) debido a la confiabilidad y veracidad con relación a la información obtenida (González et al., 2014).

Para el cálculo de los márgenes de comercialización se definieron como componentes de los costos de comercialización (CC) incurridos por los diferentes agentes participantes en el proceso: mano de obra directa, transporte, gastos administrativos, entre otros, se calculó costos fijos y variables en la comercialización de leche, dividiéndolos entre la cantidad de litros comercializados, obteniendo el costo de comercialización por litro, valor que se utilizó para el cálculo en las fórmulas.

Para determinar el costo de mano de obra al intermediario se le asignó una retribución económica por su trabajo, igual al promedio del jornal pagado en la zona (10.00 USD).

\section{Resultados y Discusión}

\section{Productores}

El $77 \%$ de los productores de la parroquia Guasaganda explotan ganado doble propósito, el 19\% tiene ganado lechero y apenas el 4\% de ellos explota ganado de carne. La mayor parte de los productores pequeños $\left(70.37 \%\right.$ ) producen un promedio de $3 \mathrm{~L} \mathrm{vaca}^{-}$ 1 día ${ }^{-1}$; los productores medianos (54.72) y grandes $(51.11 \%)$ producen en promedio $8 \mathrm{~L} \mathrm{vaca}^{-1} \mathrm{día}^{-1}$, con rangos que van de 6 a 12 litros.

Los predios de los productores pequeños tienen en su mayoría una superficie de 1 a 80 ha; mientras que los medianos (69\%) y los grandes (100\%) tienen más de 80 hectáreas.

Entre las razas que explotan los productores de la parroquia Guasaganda, la más representativa es la Holstein $(45 ; 35$ y 70\% para pequeños, medianos y grandes productores, en su orden). Además, los pequeños y medianos productores explotan las razas Gyr (20 y 30\%, respectivamente) y Jersey (15 y 25\%, respectivamente).

Los ganaderos medianos (75\%) y grandes (100\%) llevan registros de producción, genéticos, productivos, reproductivos, sanitarios, ingresos y egresos dentro de su explotación, los ganaderos pequeños (90\%) 
solo registran producción, ingresos y egresos. La identificación y registros individuales permiten un seguimiento reproductivo y productivo por vaca, lo que favorece el resultado de la explotación y el desempeño reproductivo (Silva et al., 2004).

En este estudio se observó que en los pequeños y medianos productores el tipo de mano de obra que predomina es la familiar con un $80 \%$, característica que coincide con la tipificación del sistema familiar reportado por Romo et al., 2014, donde se observó que un $85.40 \%$ de la unidades de producción de leche en el Altiplano Central mexicano utilizan generalmente a familiares directos del productor; el porcentaje restante utiliza jornaleros de la zona contratados por semana y no tienen parentesco con el productor (Martínez et al., 2012a). La remuneración diaria promedio corresponde a 8 USD si es familiar y 10 USD si son jornaleros, recibiendo en ambos casos su remuneración cada semana.

La parroquia Guasanda produce anualmente 1254,416.80 litros de leche cruda, donde los grandes productores aportan el $55.80 \%$ de dicha producción, los medianos el $38.50 \%$ y los pequeños contribuyen con un 5.70 por ciento.

En cuanto a los costos de producción de un litro de leche se realizaron los cálculos considerando un promedio de producción de 8 litros $v \operatorname{vac}^{-1}$ día $^{-1}$ para los medianos y grandes productores y 3 litros vaca ${ }^{-1}$ día $^{-1}$ para los pequeños productores, a un precio de venta en la finca de 0.30 dólares el litro, con esos valores el costo de producción obtenido fue $0.22 ; 0.20$ y 0.18 dólares por cada litro de leche producido por los pequeños, medianos y grandes productores, respectivamente; y la rentabilidad que se generó en cada estrato de productores fue 37 ; 49 y 66 por ciento (Cuadro 2).

Cuadro 2. Costos de producción de un litro de leche a nivel de pequeños, medianos y grandes productores

\begin{tabular}{lrrr}
\hline \multirow{2}{*}{ Rubros } & \multicolumn{3}{c}{ Productores } \\
\cline { 2 - 4 } & Pequeños & Medianos & Grandes \\
\hline Ingresos (USD) & 409.50 & 2788.80 & 4038.72 \\
Egresos (USD) & 299.22 & 1876.93 & 2430.96 \\
Beneficio neto (USD) & 110.28 & 911.87 & 1607.76 \\
Rentabilidad (\%) & 37.00 & 49.00 & 66.00 \\
Costo (L) (USD) & 0.22 & 0.20 & 0.18 \\
\hline
\end{tabular}

\section{Canales de comercialización}

Se observaron dos canales de comercialización: nivel cero, o canal directo de mercado (García et al., 2007), el mismo que consiste en la venta directa del productor al consumidor final y se da solo en los pequeños productores de la parroquia Guasaganda, coincidiendo con Trejo-Tellés et al. (2011), quienes indican que la utilización de canales cortos es típica de sistemas tradicionales con predominio de relaciones comerciales informales y se orienta a nichos de mercado, este canal le permite al productor obtener un mejor precio que el pagado por el intermediario o acopiador rural, sin embargo, él debe enfrentar los costos y riesgos de transporte y almacenamiento (Chauvet, 1990). El canal largo o tradicional de cinco niveles contempla igual número de intermediarios de ventas: 1) Productor - Acopiador rural - industria pasteurizadora - detallista - consumidor final, 2) Productor - Acopiador rural - industria artesanal detallista - consumidor final. Es importante indicar que la relación entre productor (pequeño y mediano) y acopiador rural no está formalizada mediante contrato, mantienen un compromiso de palabra, lo cual permite que en cualquier momento se pueda dar por terminado el compromiso adquirido (Espinoza et al., 2008). Este tipo de procedimiento permite tanto al productor como al intermediario ajustarse a las variaciones de mercado. Por el contrario, el productor grande si firma contrato para la entrega de leche cruda a la industria pasteurizadora que comercializa su producto, donde se pacta precios y volúmenes de entrega entre otras condiciones.

Los canales de comercialización identificados en la parroquia Guasaganda, permiten dinamizar la economía de mercado (Meléndez y Baños, 1984), ya que su principal función es la de controlar la distribución de bienes y servicios, especialmente en el intercambio de derecho entre el productor y el consumidor final (Espinoza et al., 2008).

Los pequeños y medianos productores realizan la venta de leche en sus predios, dejando hasta un $20 \%$ de la producción para autoconsumo, producción de derivados y alimentación de terneros, difiriendo con el MAGAP (2008), quienes aseguran que el uso y destino de la leche en el país tiene un comportamiento regular y sus estimaciones indican que entre el 25 y $32 \%$ de la producción está destinada a los fines antes descritos. Cuando por alguna razón los acopiadores no llegan a la finca a recolectar la leche los pequeños y medianos productores elaboran queso, de esta manera evitan la pérdida del producto.

Los grandes productores por su parte, venden el $100 \%$ de la producción y registran un $2 \%$ de mermas, este estrato de productores utilizan sustituto de leche para la alimentación de terneros, por lo que resulta explicable el $100 \%$ de la venta de su producción.

Con respecto a la comercialización, un $60 \%$ de los productores pequeños entregan la producción a 
intermediarios (en sus predios) y el porcentaje restante realiza la venta directa de la leche cruda al consumidor final. Los medianos productores venden la leche cruda a industrias artesanales de la localidad que producen queso y los grandes productores en un 50\% entregan la producción a industrias pasteurizadoras de la zona y el porcentaje restante lo hace a los acopiadores rurales quienes entregan el producto a las empresas de la localidad que producen queso (Figura 2).

$\mathrm{El}$ acopiador rural recoge el producto en las fincas de los productores, actividad que realiza todos los días y en horas de la mañana con la finalidad de no tener pérdidas del producto no temperado, lo cual es una estrategia para no invertir en la conservación de la leche.

El hecho de que el acopiador recoja la leche solamente por las mañanas explica la racionalidad de los productores para realizar un ordeño diario, pues si realizaran un segundo ordeño en la tarde, le correspondería asumir los riesgos de la conservación del producto, implicaría realizar inversiones para comprar equipos de refrigeración y otros de manejo, este comportamiento también permite explicar el porqué de no incrementar el volumen de producción por parte de los productores, además de evidenciar la desorganización, característica reflejada en este tipo de productores (Pastrana; citado por Espinoza et al., 2002).

Los productores reciben semanalmente el pago por su producto, cada productor y acopiador registran a diario los litros entregados y recibidos respectivamente, actividad que realizan como una medida de control (Martínez et al., 2012b), las anotaciones se registran en una libreta de campo esto pone en evidencia que los acopiadores trabajan sin invertir capital propio, característica que le permite al productor solicitar anticipo de dinero cuando tiene alguna emergencia y al intermediario lo compromete a facilitar el dinero requerido sin cobrar intereses por ello. Este comportamiento permite deducir la importancia que tiene el acopiador o intermediario dentro de los canales de comercialización de la parroquia Guasaganda, pues además de comprar el producto (leche) muchas veces sirve como apoyo económico al productor, dándole la liquidez que las instituciones financieras no le brindan ó no tienen acceso, ya sea por falta de conocimientos para acceder a créditos o tiempo para realizar los trámites.

\section{Producción de derivados lácteos a nivel artesanal}

El eslabón industrial de la cadena láctea, lo componen todos los derivados directos de la leche, obtenidos de la transformación que de dicho insumo hacen los procesadores (MADR, 2003), en la parroquia Guasaganda, el principal derivado lácteo que se produce es el queso, para ello adquieren el producto (leche cruda) diariamente en las fincas de los productores o en su defecto al acopiador rural, de los cuales Comercial Junior adquiere la mayor cantidad de leche $(78,000$ $\mathrm{L}$ año ${ }^{-1}$ ), lo que corresponde al $33.19 \%$ del total de compra de las queserías $\left(235,040 \mathrm{~L}\right.$ año $\left.0^{-1}\right)$. Estas microempresas se dedican a elaborar quesos en bloques de $10 \mathrm{~kg}(78.62 \%)$ y por libra (21.38\%) los cuales son comercializados en los mercados de La Maná, Quevedo, San Carlos y Mocache. El precio promedio por kg, al cual es introducido en el mercado el queso es 3.52 dólares.

\section{Márgenes de comercialización de leche fluida $\mathbf{y}$ queso}

Los acopiadores rurales de la zona de estudio acopian leche a un total de 107 productores comercializando un total de 608,000 litros de leche al año a un precio promedio de 0.30 dólares cada litro pagado al productor (Cuadro 3).

Los precios promedios de venta alcanzados por los diferentes actores participantes en el proceso de

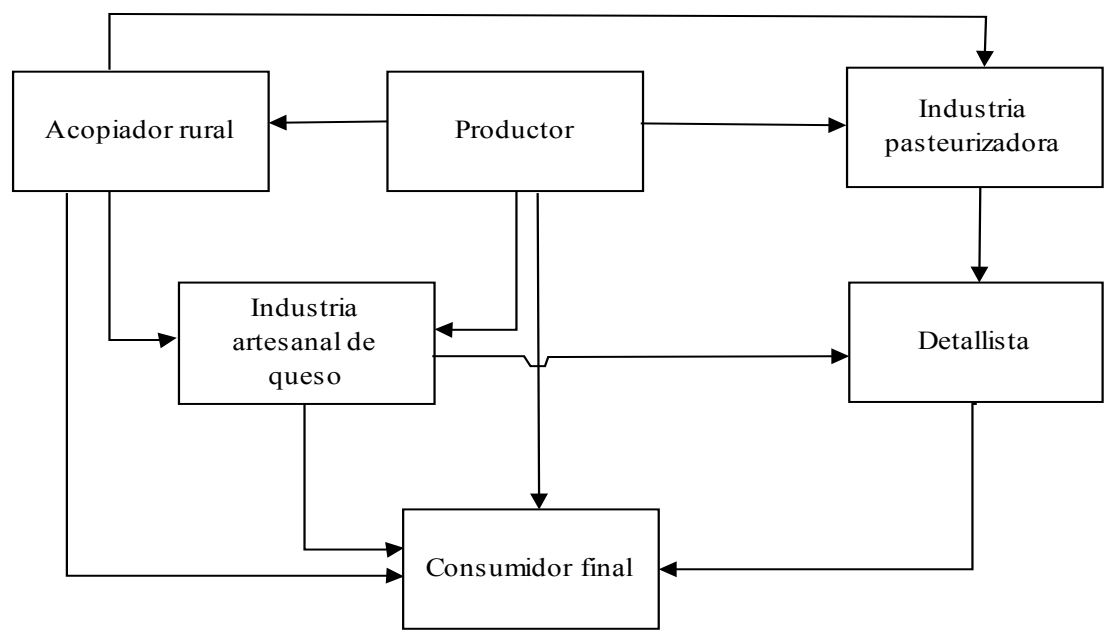

Figura 2. Canales de comercialización de la leche bovina y sus derivados 
comercialización en la zona de estudio durante el 2011 correspondieron para el productor 0.30 USD/ litro, acopiador rural $0.42 \mathrm{USD} /$ litro, mientras que la industria pasteurizadora obtuvo un precio de 0.70 USD y, el detallista vendió al consumidor final a 0.75 USD el litro de leche.

Cuadro 3. Acopiadores, volúmenes comercializados al año y precio pagado al productor de leche

\begin{tabular}{cccc}
\hline Acopiador & Productores & $\begin{array}{c}\text { Litros } \\
\text { comercializados } \\
\text { al año }\end{array}$ & $\begin{array}{c}\text { Precio } \\
\text { pagado al } \\
\text { productor }\end{array}$ \\
\hline 1 & 40 & 78 & 0.30 \\
2 & 22 & 90 & 0.30 \\
3 & 12 & 130 & 0.30 \\
4 & 18 & 130 & 0.30 \\
5 & 15 & 180 & 0.30 \\
\hline Total & $\mathbf{1 0 7}$ & $\mathbf{6 0 8}$ & \\
\hline
\end{tabular}

El margen bruto de comercialización (MBC) de leche fluida resultó en $60 \%$ lo cual indica que por cada dólar pagado por el consumidor, 0.60 USD correspondió a la intermediación. Realizando los cálculos correspondientes el MNC resultó ser 38.7\% (se consideró un costo de comercialización de 0.11 USD por litro). La participación del productor (PDP), es decir, la porción del precio pagado por el consumidor final que corresponde al productor es de 40\%. La participación de la intermediación se distribuye de la siguiente manera: al acopiador rural le corresponde el 16\%, a la industria pasteurizadora el $37.3 \%$ y al detallista el 6.7 por ciento. En cuanto a la industria artesanal de queso los precios de venta alcanzados para el productor fue de 2.10 USD por $\mathrm{kg}$, considerando que el acopiador rural es propietario de la industria artesanal de queso, por lo tanto compra al productor a 0.30 USD el litro, el costo de transporte y manipulación de la leche corresponde a 0.07 USD por litro y se consideró 7 litros de leche para producir $1 \mathrm{~kg}$ de queso. La industria artesanal vendió a 2.59 USD por $\mathrm{kg}$, mientras que el detallista obtuvo un precio de 3.19 USD y vendió al consumidor final a 3.52 USD cada kilogramo de queso comercializado.

El margen bruto de comercialización (MBC) de queso resultó en $40.34 \%$ lo cual indica que por cada dólar pagado por el consumidor, 0.403 USD correspondió a la intermediación. Realizando los cálculos correspondientes el MNC resultó ser 29.8\% (se consideró un costo de transformación y comercialización de 0.37 USD por $\mathrm{kg}$ de queso). La participación del productor (PDP), es decir, la porción del precio pagado por el consumidor final que corresponde al productor fue de 59.66\%. La participación de la intermediación se distribuye de la siguiente manera: a la industria artesanal le correspondió el $13.92 \%$ y al detallista el 26.42 por ciento.

\section{Conclusiones}

$\mathrm{E}^{\mathrm{n}}$ n la parroquia Guasaganda la actividad ganadera es muy importante desde el punto de vista socioeconómico ya que representa varios beneficios entre los que sobresalen los siguientes: 1) Generador de empleo al pequeño y mediano productor y su familia, 2) Participación importante en la seguridad alimentaria de la población, al proveer alimento de alta calidad nutritiva, 3) Dinamizador de la economía de la zona al ser generador de empleo permanente y fuente de subsistencia para muchas familias y áreas aledañas, 4) Abastecedor de materia prima de calidad para la pequeña y gran industria $\mathrm{y}, 5$ ) Mejora los niveles de vida de los productores y su sentido de pertenencia hacia la actividad ganadera.

El entendimiento de los canales y márgenes de comercialización permitirá que las utilidades generadas se repartan de manera equitativa entre el productor y el acopiador para satisfacer las necesidades socioeconómicas de la población colocando al alcance de ésta un producto con excelente valor nutricional y menor precio.

El impulso por parte del gobierno nacional al cambio de la matriz productiva permite vislumbrar externalidades positivas tanto a nivel nutricional como en los canales y agentes de comercialización identificados, para concientizar a los ganaderos en la importancia de producir leche de calidad, que les permita obtener mejores precios, mayor demanda, lo que estimulará a los productores a participar activamente en sus respectivas organizaciones, para obtener beneficios como tecnificación, créditos, capacitación, compra de insumos y venta asociativa de la leche.

\section{Bibliografía}

Chauvet, S.M. 1990. ¿La ganadería nacional en vías de extinción?. México. Comercio Exterior 40(9): 35.

Espinosa, V., Rivera, G., García, L. 2008. Los canales y márgenes de comercialización de la leche cruda producida en sistema familiar (Estudio de caso). Vet. Méx 39(1):1-16.

Espinoza, V., López, C., García, G., Gómez, L., Velásquez, P., Rivera, G. 2002. Márgenes de comercialización de la leche cruda producida en sistema familiar. Revista Científica 12(Supl. 2): 650-654.

FAO (Organización de las Naciones Unidas para la 
Agricultura y la Alimentación). 1999. Cumbre Mundial sobre la alimentación. Roma-Italia. (en línea). Consultado el 12 de octubre de 2013. Disponible en: http:/www.fao.org/wfs/index es.htm.

FAO y FIL. 2012. Guía de buenas prácticas en explotaciones lecheras. Directrices FAO: Producción y Sanidad Animal, No. 8. Roma.

FAO-FEPALE. 2012. Situación de la Lechería en América Latina y el Caribe en 2011, Observatorio de la Cadena Lechera. Oficina Regional de la FAO para América Latina y el Caribe, División de Producción y Sanidad Animal. Chile. 70 p.

García, A., Perea, J. Acero, R., Valerio, D., Rodríguez, Gómez, V. 2007. Circuito de comercialización de la leche ecológica en siete comunidades autónomas españolas. Arch. Zootec. 56(Supl. 1): 693-698.

García, L., Aguilar, A., Luévano A., Cabral, A. 2005. Globalización productiva y comercialización de la leche y sus derivados: Articulación de la ganadería intensiva lechera de la Comarca Lagunera. Plaza y Valdez. México. 278 p.

González, F., Rebollar, S., Hernández, J., Guzmán, E. 2014. La Comercialización de la miel en el Sur del Estado de México. Revista Mexicana de Agronegocios 18(34): 806-815.

MADR. (Ministerio de Agricultura y Desarrollo Rural). 2003. Observatorio Agrocadenas Colombia.La Cadena Láctea en Colombia. Documento de trabajo No. 4. Bogotá: MADR. (en línea). Disponible en: http://www.fedeleche.cl/infotec/leche-colombia. pdf.

MAGAP. (Ministerio de Agricultura, Ganadería, Acuacultura y Pesca). 2008. Cadena consultiva de la leche, Terminales y servicios. (en línea). Consultado 5 nov 2008. Disponible en www. magap.gov

Martínez, C.J., Cotera, J., Abad, J. $2012 \mathrm{a}$. Comercialización de leche bovina en sistemas de doble propósito en Dobladero, Veracruz. Quinta Época 30: 816-824.

Martínez, C.J., Cotera, J., Abad, J. 2012b. Características de la producción y comercialización de leche bovina en sistemas de doble propósito en Dobladero, Veracruz. Revista Mexica de Agronegocios, 16(30): 816-824

Meléndez, G.R., Baños, C.A. 1984. Mercadeo de productos agropecuarios. 1ra edición Limusa. México. 43-51.

Padilla, N. 2005. El acuerdo de complementación económica. No. 59 CAN-MERCOSUR en la producción y comercialización de leche en ecuador. Tesis Ingeniero en Comercio Exterior e integración. UTE. Quito. 293 p.

Romo, C., Valdivia, A., Carranza R., Cámara, J. Zavala, M., Flores, E., Espinosa, J. 2014. Brechas de rentabilidad económica en pequeñas unidades de producción de leche en el antiplano central mexicano. Rev. Mex. Ciencias Pecuarias 5(3): 273-290.

Santoyo-Cortés, H., Ramírez-Moreno, P., Suvedi, M. 2000. Manual para la evaluación de programas de desarrollo rural. Centro de Investigaciones Económicas, Sociales y Tecnológicas de la Agroindustria y laAgricultura Mundial-Universidad Autónoma Chapingo-Instituto Nacional de Capacitación Rural. México, D.F. 250 p.

SICA-MAGAP (Servicio de Información y Censo Agropecuario). 2000. Proyecto SICA-MAGAP. Perspectiva de la producción láctea en el Ecuador. (en línea). Consultado 5 enero 2014. Disponible en: www.sica.com.

Silva, D., Peña, M.E., Urdaneta, F. 2004. Registros de control e indicadores de resultados en ganadería de doble propósito. Revista Científica FCV-LUZ. 20(1): 89-100

Torres, E., Quisphe, D., Sánchez, A., Reyes, M., González, B., Torres, A., Cedeño, A., Haro, A. 2013. Caracterización de la producción de frijol en la provincia de Cotopaxi Ecuador: caso comuna Panyatug. Ciencia y Tecnología 6(1): 23-31.

Torres, Y., Rivas, J., De Pablos-Heredero, C., Perea, J., Toro-Mujica, P., Angón, E., García, A. 2014. Identificación e implementación de paquetes tecnológicos en ganadería vacuna de doble propósito. Caso Manabí-Ecuador. Revista Mexicana de Ciencias Pecuarias 5(4): 393-407.

Trejo-Telléz, B., de los Ríos-Carmenado, I., FigueroaSandoval, B., Gallego-Moreno, F., Morales-Flores, F. 2011. Análisis de la cadena de valor del queso Manchego en Cuenca. España. Revista Mexicana de Ciencias Agrícolas 2(4): 545-557. 